\title{
Monitoring the depth of anaesthesia using the new modified entropy sensors during supratentorial craniotomy: Our experience
}

\author{
Richa Sharma, Pirjo Manninen, Lashmikumar Venkatraghavan
}

\begin{abstract}
Background: Monitoring the depth of anaesthesia can be a challenge in patients undergoing supratentorial craniotomy because the conventional sensors for both bispectral index and entropy monitors lose their contact with a brain after scalp elevation. The new sensors for the entropy monitor are more flexible and can be placed in different locations. The purpose of this study was to determine the feasibility on the use of new GE entropy sensors in monitoring depth of anaesthesia in patients undergoing supratentorial craniotomy. Materials and Methods: We retrospectively reviewed the data from 20 consecutive patients undergoing supratentorial craniotomy who had the monitoring of the depth of anaesthesia using modified entropy sensors. Prior to the induction of anaesthesia, the new GE entropy sensor (P/N MI03868I) was applied in a modified fashion. We measured the state entropy (SE) and response entropy (RE) at 12 perioperative time points. Entropy values were compared with the clinical indices of depth of anaesthesia. Results: Data from 20 consecutive patients (orbitozygomatic craniotomy [10] and bifrontal craniotomy [10]) were analysed. Monitoring was possible in all the patients. The changes in entropy values correlated with clinical indices of depth of anaesthesia. However, some patients showed variations in absolute values (RE and SE) during the intraoperative period without any changes in the level of anaesthetic depth. Conclusions: Monitoring the depth of anaesthesia is feasible with the use of new entropy sensors in patients undergoing supratentorial craniotomy. In contrast to standard sensors, the new sensors offer flexibility with the placement.
\end{abstract}

Key words: Bispectral index, depth of anaesthesia, entropy, neurosurgery

\section{INTRODUCTION}

Electroencephalography (EEG) based depth of anaesthesia monitors are commonly used to assist titration of anaesthetic-hypnotic agents. These devices have been shown to decrease intraoperative anaesthetic usage, improve intraoperative haemodynamics, and promote faster recovery. ${ }^{[1]}$ However, the use of

\begin{tabular}{|l|l|}
\hline \multicolumn{2}{|c|}{ Access this article online } \\
\hline Quick Response Code: & Website: \\
\hline & www.jnaccjournal.org \\
\cline { 2 - 2 } & \\
\hline
\end{tabular}

depth of anaesthesia monitors in patients undergoing neurosurgery, particularly the supratentorial craniotomy, can be a challenge from both surgical and anaesthetic perspectives. The conventional sensors for both bispectral index (BIS) (Aspect Medical Inc., Newton, MA, USA) and Entropy (GE Healthcare, Helsinki, Finland) monitors have strip sensors that are placed in a bifrontal distribution, interfering considerably with the surgical field and scalp incision. Furthermore, after scalp elevation, the montage system loses brain contact resulting in poor signal transference. ${ }^{[2-4]}$ Studies have looked into the alternate placements of sensors namely occipital and nasal montages, but the results are inconclusive. ${ }^{[2-7]}$ The new GE entropy sensors $(\mathrm{P} / \mathrm{N}$ M1038681) have three separate electrodes instead of conventional strip sensors there are three electrodes in one strip [Figure 1]. These are more flexible and can be

Department of Anesthesia, University of Toronto, Toronto Western Hospital, University Health Network, Toronto, Ontario, Canada

Address for correspondence:

Dr. Lakshmikumar Venkatraghavan, Department of Anesthesia, Toronto Western Hospital, 399, Bathurst Street, Toronto, Ontario M5T 2S8, Canada. E-mail: Lashmi.Venkatraghavan@uhn.ca 
placed in different locations and distance between the electrodes can be varied. The purpose of our study is to determine the feasibility of new GE entropy sensors in monitoring depth of anaesthesia for patients undergoing supratentorial craniotomy.

\section{SUBJECTS AND METHODS}

After institutional ethics board approval, we retrospectively reviewed the data from 20 consecutive patients undergoing supratentorial craniotomy who had depth of anaesthesia monitoring using modified entropy sensors. Ten patients underwent orbitozygomatic craniotomy, and the remaining had bifrontal craniotomy. Prior to the induction of anaesthesia, the new GE entropy sensor (P/N M1038681) was applied in a modified fashion with three sensors placed on the ipsilateral frontal, temporal and maxillary bones respectively [Figures 2 and 3]. For patients undergoing orbitozygomatic craniotomy, the sensors were placed on the opposite side of the face. Anaesthesia management was standard in all patients and consisted of propofol, fentanyl and rocuronium for induction. Oxygen and air, with either sevoflurane or desflurane, was employed for maintenance of anaesthesia. We measured the state entropy (SE) and response entropy (RE) at 12 perioperative time points including awake, loss of eyelash reflex, intubation, knife to skin intraoperative time one, intraoperative time two, scalp closure commencement, scalp closure completion, anaesthetic agent off, obeying commands, extubation, and postextubation. Other data collected include patient demographics, surgical procedure, anaesthetic data, and the intraoperative haemodynamics.

\section{RESULTS}

Data from 20 consecutive patients (mean age of 47 years, 12 males and 8 females) were analysed. Monitoring was possible in all the patients. In three patients, electrode position was changed during the head pinning as the electrodes were at the pin site. The entropy (RE and $\mathrm{SE})$ values of all the patients at various perioperative points are shown in Figure 4. Overall the changes in entropy values correlated with clinical indices of depth of anaesthesia (hemodynamic and end-tidal anaesthetic agent concentration) [Tables 1 and 2]. However, in some of the patients, there were unexplained increases in entropy values (RE and SE) during the intraoperative period without any changes in the level of anaesthetic depth or surgical stimulation [Figure 4].

\section{DISCUSSION}

Our case series has shown that the newer GE entropy sensors (P/N M10338681) may represent a feasible option for consistent entropy monitoring in patients undergoing procedures in which standard bifrontal montages present considerable inconvenience and

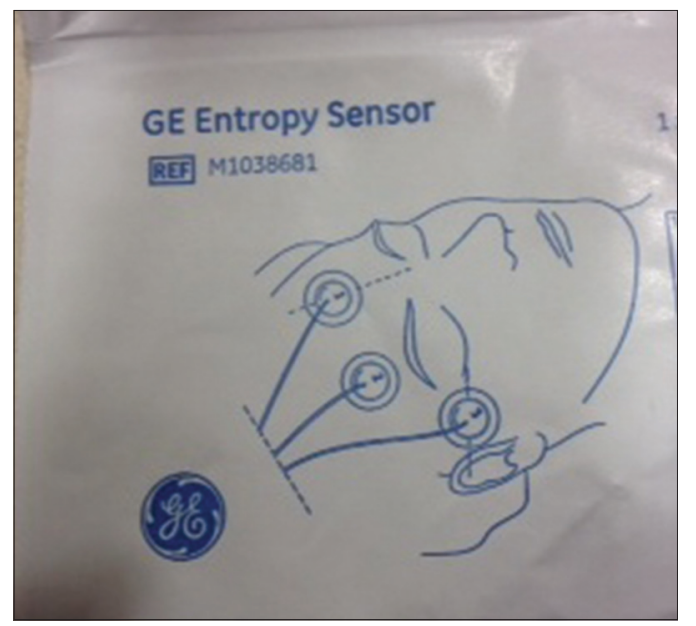

Figure 1: The new GE entropy sensor (P/N M10338681) and the manufacturer recommended placement of the new GE entropy sensor

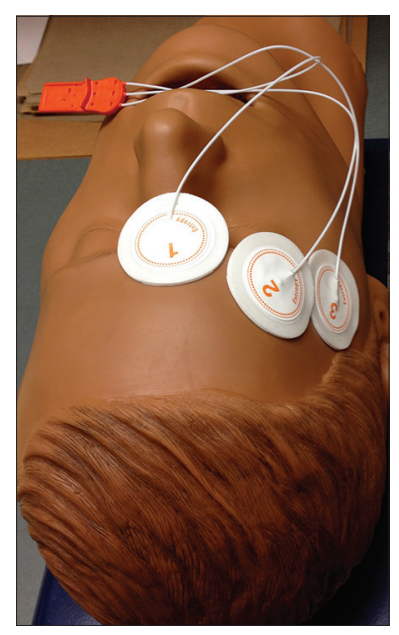

Figure 2: The study placement of new GE entropy electrode unilateral placement

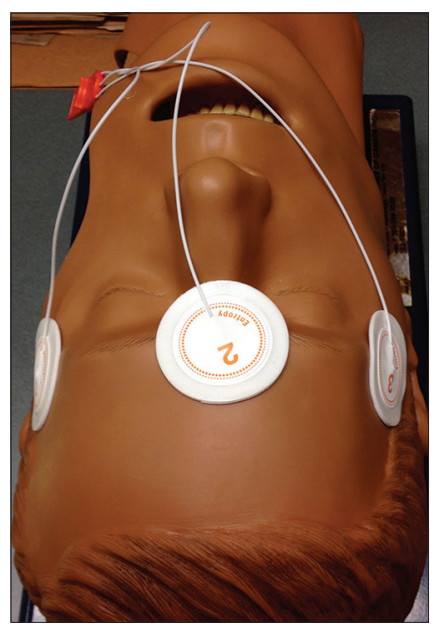

Figure 3: The study placement of new GE entropy electrode bilateral placement 


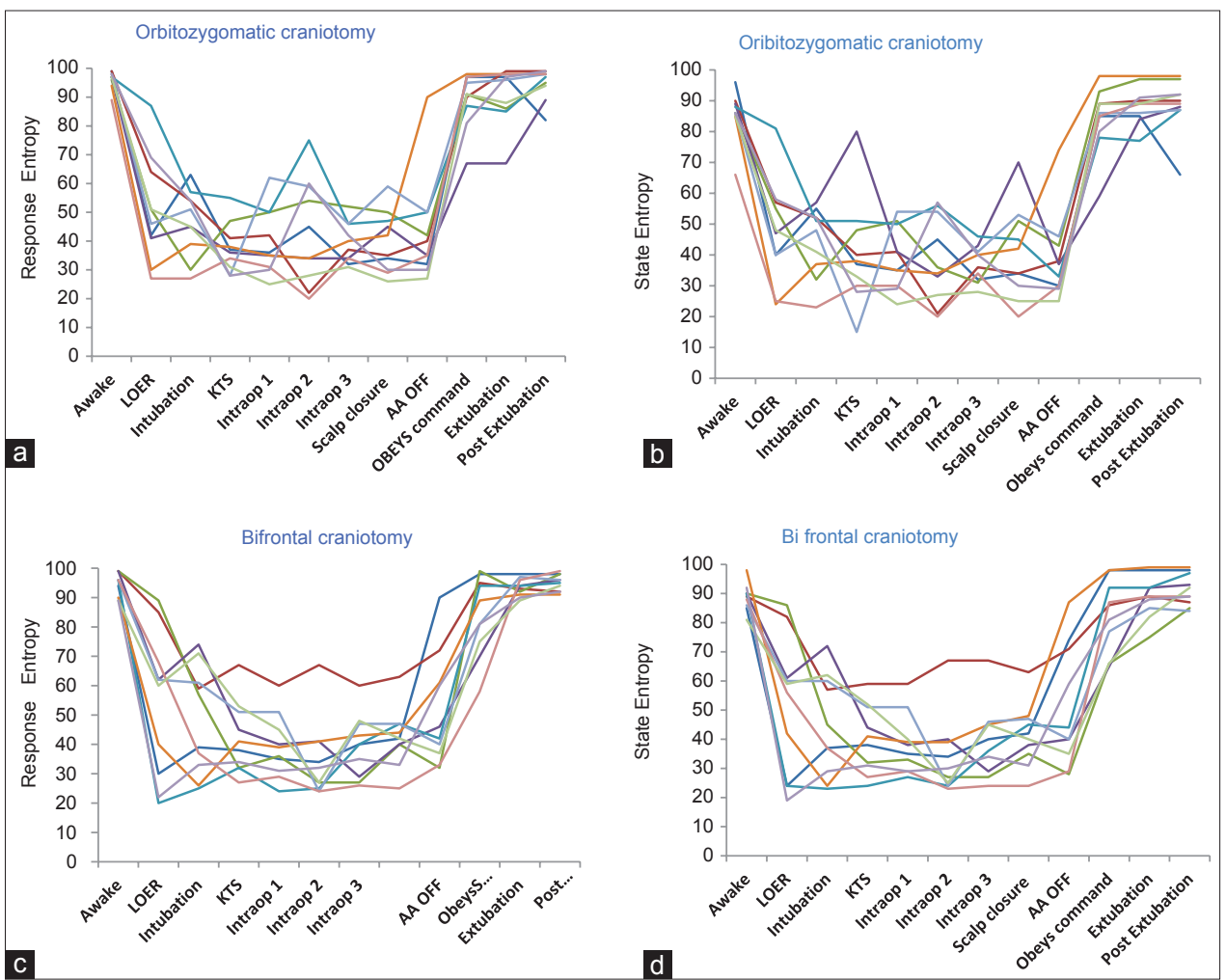

Figure 4: The line diagrams of response entropy and state entropy values during different stages of anesthesia in patients undergoing orbitozygomatic and bifrontal craniotomies. Each line represents an individual patient. LOER: Loss of eyelash reflex. KTS: Knife to skin. AA: Anesthetic agent

Table 1: Entropy values and MAC levels at critical time points in patients undergoing orbitozygomatic craniotomy

\begin{tabular}{|c|c|c|c|c|c|c|c|c|c|c|c|c|}
\hline \multirow{2}{*}{$\begin{array}{l}\text { Patient } \\
\text { number }\end{array}$} & \multicolumn{2}{|c|}{ Induction } & \multicolumn{2}{|c|}{ Knife to skin } & \multicolumn{2}{|c|}{ Intraoperative 1} & \multicolumn{2}{|c|}{ Intraoperative 2} & \multicolumn{2}{|c|}{ Intraoperative 3} & \multicolumn{2}{|c|}{ Extubation } \\
\hline & $\mathrm{RE}^{*}$ & $\mathrm{MAC}^{\dagger}$ & RE & MAC & RE & MAC & RE & MAC & RE & MAC & RE & MAC \\
\hline 1 & 99 & 0 & 37 & 1.1 & 36 & 1.1 & 45 & 1.1 & 32 & 1.1 & 97 & 0.1 \\
\hline 2 & 99 & 0 & 41 & 0.9 & 42 & 1 & 22 & 1 & 37 & 1.1 & 99 & 0 \\
\hline 3 & 96 & 0 & 47 & 0.7 & 50 & 0.7 & 54 & 0.7 & 52 & 0.7 & 86 & 0 \\
\hline 4 & 97 & 0 & 36 & 1 & 35 & 2 & 34 & 1.9 & 34 & 1.9 & 67 & 0.1 \\
\hline 5 & 97 & 0 & 55 & 1.1 & 50 & 1.1 & 75 & 1.5 & 46 & 1 & 85 & 0.2 \\
\hline 6 & 94 & 0 & 38 & 1 & 35 & 0.9 & 34 & 0.9 & 40 & 0.9 & 98 & 0.1 \\
\hline 7 & 98 & 0 & 28 & 0.7 & 62 & 0.7 & 59 & 0.7 & 46 & 0.6 & 96 & 0.1 \\
\hline 8 & 89 & 0 & 34 & 0.7 & 31 & 0.8 & 20 & 0.9 & 34 & 0.7 & 98 & 0 \\
\hline 9 & 97 & 0 & 31 & 0.8 & 25 & 1 & 28 & 1 & 31 & 1 & 88 & 0.1 \\
\hline 10 & 98 & 0 & 28 & 0.4 & 30 & 1 & 60 & 1 & 42 & 0.9 & 97 & 0.1 \\
\hline
\end{tabular}

questionable reliability. Our results show a stable trend in RE and SE values that are clinically consistent with appropriate anaesthetic depth. In contrast to standard sensors (strip), the new sensors offer flexibility with respect to the placement.

The basic principle of scalp EEG recordings is based on the principle of measuring the field potentials. ${ }^{[8]}$ EEG electrodes do not measure the action potential of individual neurons. Instead, they measure the postsynaptic potentials of a group of underlying neurons. These potentials are recorded via scalp electrodes, providing a map of electrical activity of the brain. Since the field potentials are the overall sum of the postsynaptic potentials occurring at distance from the recording sites, the placement of montages does not need to be very precise. In the intraoperative setting, a few centimetres of displacement (resulting from surgical 
Table 2: Entropy values and MAC levels for critical time points in patients undergoing bifrontal craniotomy

\begin{tabular}{|c|c|c|c|c|c|c|c|c|c|c|c|c|}
\hline \multirow{2}{*}{$\begin{array}{l}\text { Patient } \\
\text { number }\end{array}$} & \multicolumn{2}{|c|}{ Induction } & \multicolumn{2}{|c|}{ Knife to skin } & \multicolumn{2}{|c|}{ Intraoperative 1} & \multicolumn{2}{|c|}{ Intraoperative 2} & \multicolumn{2}{|c|}{ Intraoperative 3} & \multicolumn{2}{|c|}{ Extubation } \\
\hline & $\mathrm{RE}^{*}$ & $\mathbf{M A C}^{\dagger}$ & RE & MAC & RE & MAC & RE & MAC & RE & MAC & RE & MAC \\
\hline 1 & 94 & 0 & 38 & 1 & 35 & 0.9 & 34 & 0.9 & 40 & 0.9 & 98 & 0.1 \\
\hline 2 & 99 & 0 & 67 & 1 & 60 & 1 & 67 & 1 & 60 & 1 & 93 & 0 \\
\hline 3 & 99 & 0 & 32 & 1 & 36 & 1 & 27 & 1.1 & 27 & 1.1 & 92 & 0 \\
\hline 4 & 99 & 0 & 45 & 0.6 & 40 & 1 & 41 & 1 & 29 & 1 & 94 & 0 \\
\hline 5 & 96 & 0 & 32 & 0.8 & 24 & 1.2 & 25 & 1.2 & 40 & 0.9 & 94 & 0 \\
\hline 6 & 90 & 0 & 41 & 0.9 & 39 & 0.9 & 41 & 0.9 & 43 & 0.9 & 91 & 0 \\
\hline 7 & 96 & 0 & 51 & 0 & 51 & 1.1 & 24 & 1.1 & 47 & 1.1 & 97 & 0 \\
\hline 8 & 68 & 0 & 27 & 0.7 & 29 & 1 & 24 & 1 & 26 & 1 & 96 & 0.2 \\
\hline 9 & 89 & 0 & 53 & 0.9 & 45 & 0.9 & 27 & 0.9 & 48 & 0.8 & 89 & 0 \\
\hline 10 & 89 & 0 & 34 & 0.9 & 31 & 0.9 & 32 & 0.9 & 35 & 0.8 & 90 & 0 \\
\hline
\end{tabular}

field interference) is usually acceptable in achieving signals for the placement of a variety of montages including EEG, somatosensory evoked potential, and motor evoked potential.

Standard sensors can be moved to one side of the forehead, instead of the bifrontal location, and have been shown to provide reliable indices of depth of anaesthesia. ${ }^{[5-7]}$ Current practice in many centres is to place the standard sensors unilaterally in patients undergoing supratentorial craniotomy. However, the three individual electrodes in the standard sensors are in a fixed position, thus making modification of their location difficult. In the new entropy sensors, the three electrodes are free, providing more flexibility with placement. A comparison study conducted by GE in 2009 showed no statistically significant difference between $\mathrm{SE}$ and RE values, EEG signal, interference tolerance, electrode impedance, adhesive performance, and overall usability between the standard GE Entropy Easyfit sensor and the new GE entropy sensors. ${ }^{[9]}$

Originally, all the processed EEG devices were designed and validated using frontal montages (Fpz, F7). Theoretically, if the electrodes are displaced only few centimetres away from the suggested frontal position, the electrodes still measure the same underlying cerebral cortex. On the other hand, if we use the montages in a totally different area (e.g., occipital lobe) to replace the frontal montages, we are assuming the raw EEG signals in different lobes would behave the same fashion in frontal lobes. Use of different BIS-vista montages has been examined in previous studies. The use of the fronto-central placement was found to differ in an unpredictable manner. ${ }^{[4]}$ The use of occipital montages found to have poor agreement between the frontal and occipital values. ${ }^{[4,6]}$ In a recent study, Nelson et al. reported statistically significant variability, but no appreciable clinical difference, between placing the sensor over the frontal bone versus the nasal bridge. The authors recommended the nasal bridge placement for procedures in which the monitor may interfere with the surgical field. ${ }^{[7]}$ One of the major limitation in our study was that we did not compare the new sensors with that of the conventional sensors. Hence, we cannot determine the reliability of the new sensor in the modified position, and there is a need for a prospective study to determine this.

In summary, monitoring the depth of anaesthesia in neurosurgical patients undergoing supratentorial craniotomy is feasible with the use of new three electrodes entropy sensors. Our results show a stable trend in RE and SE values that are clinically consistent with appropriate anaesthetic depth. In contrast to standard sensors (strip), the new sensors offer flexibility with the placement.

\section{REFERENCES}

1. Shepherd J, Jones J, Frampton G, Bryant J, Baxter L, Cooper K. Clinical effectiveness and cost-effectiveness of depth of anaesthesia monitoring (E-Entropy, Bispectral Index and Narcotrend): A systematic review and economic evaluation. Health Technol Assess 2013;17:1-264.

2. Sinha PK, Suneel PR, Unnikrishnan KP,Smita V, Rathod RC.An alternative site for entropy sensor placement. Anesth Analg 2006;102:1291.

3. Akavipat $\mathrm{P}$, Hungsawanich $\mathrm{N}$,Jansin R.Alternativeplacementof bispectral index electrode for monitoring depth of anesthesia during neurosurgery. Acta Med Okayama 2014;68:151-5.

4. Shiraishi T, Uchino H, Sagara T, Ishii N. A comparison of frontal and occipital bispectral index values obtained during neurosurgical procedures. Anesth Analg 2004;98:1773-5.

5. Dahaba AA, Xue JX, Zhao GG, Liu QH, Xu GX, Bornemann H, et al. BIS-vista occipital montage in patients undergoing neurosurgical procedures during propofol-remifentanil anesthesia. Anesthesiology 2010;112:645-51. 
6. Hall JD, Lockwood GG. Bispectral index:Comparison of two montages. Br J Anaesth 1998;80:342-4.

7. Nelson P, Nelson JA, Chen AJ, Kofke WA. An alternative position for the BIS-Vista montage in frontal approach neurosurgical cases.J Neurosurg Anesthesiol 2013;25:135-42.

8. Simon MV. Neurophysiologic tests used in the operating room. In: Simon MV, editor. Intraoperative Clinical Neurophysiology: A Comprehensive Guide to Monitoring and Mapping. $1^{\text {st }}$ ed. New York: Demos Medical Publishing; 2010. p. 1-44.

9. Uutela K, Stebor A. GE Entropy Sensor: Comparative study.
GE Healthcare Finland, 2009. Available from: http://www. clinicalview.gehealthcare.com/download.php?obj id $=244$ and browser $=$ true. [Last cited on 2014 May 02].

How to cite this article: Sharma R, Manninen P, Venkatraghavan L. Monitoring the depth of anaesthesia using the new modified entropy sensors during supratentorial craniotomy: Our experience. J Neuroanaesthesiol Crit Care 2015;2:28-32.

Source of Support: Nil, Conflict of Interest: None declared.

\section{Author Help: Online submission of the manuscripts}

Articles can be submitted online from http://www.journalonweb.com. For online submission, the articles should be prepared in two files (first page file and article file). Images should be submitted separately.

1) First Page File:

Prepare the title page, covering letter, acknowledgement etc. using a word processor program. All information related to your identity should be included here. Use text/rtf/doc/pdf files. Do not zip the files.

2) Article File:

The main text of the article, beginning with the Abstract to References (including tables) should be in this file. Do not include any information (such as acknowledgement, your names in page headers etc.) in this file. Use text/rtt/doc/pdf files. Do not zip the files. Limit the file size to $1 \mathrm{MB}$. Do not incorporate images in the file. If file size is large, graphs can be submitted separately as images, without their being incorporated in the article file. This will reduce the size of the file.

3) Images:

Submit good quality color images. Each image should be less than $4096 \mathrm{~kb}(4 \mathrm{MB})$ in size. The size of the image can be reduced by decreasing the actual height and width of the images (keep up to about 6 inches and up to about $1800 \times 1200$ pixels). JPEG is the most suitable file format. The image quality should be good enough to judge the scientific value of the image. For the purpose of printing, always retain a good quality, high resolution image. This high resolution image should be sent to the editorial office at the time of sending a revised article.

4) Legends:

Legends for the figures/images should be included at the end of the article file. 